\title{
A new silica-infiltrated Y-TZP obtained by the sol-gel method
}

\author{
T.M.B. Campos ${ }^{\mathrm{a}}$, N.C. Ramos ${ }^{\mathrm{b}}$, J.P.B. Machado ${ }^{\mathrm{c}}$, M.A. Bottino ${ }^{\mathrm{b}}$, R.O.A. Souza ${ }^{\mathrm{d}}$, R.M. Melo ${ }^{\mathrm{b}, *}$ \\ a Instituto Tecnologico de Aeronautica (ITA), Praça Marechal Eduardo Gomes 50, 12228-900 São José dos Campos, SP, Brazil \\ b Univ. Estadual Paulista (UNESP), Institute of Science and Technology at São José dos Campos, Department of Dental Materials and Prosthodontics, Eng. \\ Francisco José Longo Avenue 777, 12245-000 São José dos Campos, SP, Brazil \\ ${ }^{\mathrm{c}}$ National Institute for Space Research, Associated Laboratory of Sensors and Materials, Astronautas Avenue 1758, 12217-010 São José dos Campos, SP, Brazil \\ ${ }^{\mathrm{d}}$ Federal University of Rio Grande do Norte (UFRN), Dentistry Department, 1787 Salgado Filho Avenue, 59056-000 Natal, RN, Brazil
}

\section{A R T I C L E I N F O}

\section{Article history:}

Received 16 October 2015

Received in revised form 3 March 2016

Accepted 9 March 2016

\section{Keywords:}

Zirconia

Silica

Sol-gel

Flexural strength

Weibull

Bond strength

\begin{abstract}
A B S T R A C T
Objective: The aim of this study was to evaluate silica infiltration into dental zirconia (VITA In-Ceram 2000 YZ, Vita Zahnfabrik) and its effects on zirconia's surface characteristics, structural homogeneity and bonding to a resin cement.

Methods: Infiltration was performed by immersion of the pre-sintered zirconia specimens in silica sols for five days (ZIn). Negative (pure zirconia specimens, ZCon-) and positive controls (specimens kept in water for 5 days, ZCon+) were also performed. After sintering, the groups were evaluated by X-ray diffraction (XRD), grazing angle X-ray diffraction (DRXR), scanning electron microscopy (SEM), contact angle measurements, optical profilometry, biaxial flexural test and shear bonding test. Weibull analysis was used to determine the Weibull modulus $(\mathrm{m})$ and characteristic strength $\left(\sigma_{0}\right)$ of all groups.

Results: There were no major changes in strength for the infiltrated group, and homogeneity $(\mathrm{m})$ was also increased. A layer of $\mathrm{ZrSiO}_{4}$ was formed on the surface. The bond strength to resin cement was improved after zirconia infiltration, acid conditioning and the use of an MDP primer.

Conclusion: The sol-gel method is an efficient and simple method to increase the homogeneity of zirconia. Infiltration also improved bonding to resin cement.

Clinical significance: The performance of a zirconia infiltrated by silica gel improved in at least two ways: structural homogeneity and bonding to resin cement. The infiltration is simple to perform and can be easily managed in a prosthesis laboratory.
\end{abstract}

(c) 2016 Elsevier Ltd. All rights reserved.

\section{Introduction}

Dental ceramics are similar to the natural tooth in appearance because of their optical properties. This and other qualities, such as hardness [1] and chemical stability [2], enabled these materials to be rapidly developed for dental use, to meet the increasing demands for esthetics and durability.

The dental ceramics presenting better esthetics are silicates like feldspathic ceramic and lithium disilicate [3]. However, these ceramics have inferior mechanical properties compared with those

\footnotetext{
* Corresponding author at: Institute of Science and Technology, Av. Engenheiro Francisco José Longo 777, 12245-200, São Dimas, São José dos Campos, SP, Brazil.

E-mail addresses: renata.marinho@ict.unesp.br, marquesdemelo@gmail.com (R.M. Melo).
}

of zirconia. Thus, zirconia has been widely used as an infrastructure material in crowns and fixed partial prostheses, because of its excellent mechanical properties [4].

The remarkable performance of zirconia, as explored in various medical and engineering applications, is due mainly to the transformation of the tetragonal into the monoclinic phase. This transformation can be induced by thermomechanical factors, resulting in a volume increase of about $3-4 \%$. This volume increase generates compressive stresses on the tip of a possible crack. In this case, for the crack to propagate, it must overcome the compressive stresses surrounding it. This toughening mechanism explains the high fracture resistance of zirconia when compared with that of other ceramics [5,6].

A common problem in zirconia is its difficulty in promoting adhesion compared with silica-based ceramics [7]. For feldspathic ceramics, conditioning the surface with hydrofluoric acid (HF 10\%) 
is effective in creating mechanical imbrication, while silanization provides the chemical bond between the inorganic ceramic and the organic resin cement. However, for alumina- and zirconia-based ceramics HF is not able to roughen the surface sufficiently, and the absence of silica prevents chemical bonding to silanes [7]. Air abrasion with aluminum oxide alone, commonly performed in laboratories, also does not improve adhesion and creates microdefects that compromise the strength of the material in the long term [8-10].

Until now, the preferred method for bonding zirconia is the combination of air abrasion with aluminum oxide particles coated with silica (silicatization) and the application of a phosphate-based monomer as an adhesion promoter. However, silicatization also has the potential to damage the surface [11-13].

Based on reports in the literature, the most frequent clinical failures of tooth- and implant-supported zirconia crowns are loss of retention and veneering material fracture [14]. To solve the retention problem, several methods were created to infuse silica into zirconia, making it chemically reactive to the bisphenol-Aglycidyl dimethacrylate (Bis-GMA)-based cements such as the PyrosilPen [15], the "glaze-on technique" [16,17], which uses a thin intermediate coating of acid-etchable glasses, and silica nanocoating [18]. Additional methods, such as selective glass infiltration [19] and a hot acidic solution [20] are intricate techniques that roughen zirconia and improve bonding. A new method for infiltrating pure silica in a Y-TZP using a sol-gel process was proposed in this present study to create a layer sensitive to acid etching and silanization.

By coating the Y-TZP we also aimed to produce a graded zirconia. This type of material presents increased load bearing capacity and a gradual increase in the elastic modulus towards the interior layers [21], which may improve the interfacial adhesion with porcelains [22].

Therefore, this first study aimed to evaluate the effects of such infiltration with a simple and low-cost sol-gel method on the mechanical properties, microstructure and surface characteristics of a Y-TZP. Additionally, we evaluated the bond strengths between the infiltrated ceramic and resin cement.

\section{Materials and methods}

\subsection{Preparation of specimens}

The pre-sintered zirconia blocks (Vita InCeram YZ, Vita Zahnfabrik, Bad Säckingen, Germany) were wet-ground into cylinders ( $18 \mathrm{~mm}$ in diameter) that were then sectioned into discs in a lathe (ISOMET 1000, Buehler, Lake Bluff, IL, USA). The first section (approximately $0.5 \mathrm{~mm}$ ) was discarded, and the remaining sections ( $1.65 \mathrm{~mm}$ thickness) were cut under coolant irrigation. The discs were wet-ground flat with silicon carbide (SiC) (\#1200) and sintered according to the manufacturer's instructions (Vita Zyrcomat, Vita Zahnfabrik) according to the following schedule: temperature elevation time, $1 \mathrm{~h}$; final temperature, $1530^{\circ} \mathrm{C}$; waiting time, $2 \mathrm{~h}$; and cooling temperature at which the tray can be downloaded inside the oven, $400^{\circ} \mathrm{C}$. The final dimensions were according to ISO 6872 standard, being $12 \mathrm{~mm}$ in diameter and $1.2 \mathrm{~mm}$ thick.

The pre-sintered zirconia discs were divided into three groups. One group was immersed in water alone (Positive Control Group, ZCon +) and/or in silica sol (ZIn), both of which remained in these respective media for $5 \mathrm{~d}$. The silica sol $(0.5 \mathrm{~mol} / \mathrm{L})$ was obtained by passing an aqueous sodium silicate solution through an ionexchanging resin. The third group was the negative control (ZCon), which was not immersed in any medium. After the immersion period, the pre-sintered zirconia discs were removed from the solution and put into an oven at $100^{\circ} \mathrm{C}$ for two days. After being dried, the specimens were sintered in the same manner as the specimens of the non-infiltrated group.

The sintered specimens were then evaluated by X-rays, Raman spectroscopy, scanning electron microscopy (SEM), and contact angle measurements. X-ray diffraction (XRD) (X'pert Powder model, PANalytical, Westborough, MA, USA) was performed at $10-90^{\circ}$, with a scan step of $10.1600 \mathrm{~s}$, at a $0.0170^{\circ}$ step size, with $\mathrm{CuK} \alpha$ radiation. The Rietveld refinement of the diffractometries was performed with the General Structure Analysis System (GSAS)/EXPGUI program (a graphical interface for the Los Alamos GSAS package). Raman spectroscopy was performed in a Micro Raman system (Renishaw 2000, Gloucestershire, UK) under Ar laser excitation, at a wavelength of $514.5 \mathrm{~nm}$. The Raman spectra were collected over 3 accumulation periods of $30 \mathrm{~s}$, between 200 and $3500 \mathrm{~cm}^{-1}$. The contact angle analysis was performed in a tensiometer (Kruss, model EasyDrop DAS 100, Hamburg, Germany) Contact Angle Measuring Instrument using the sessile drop (Young-Laplace method) and distilled water $(3 \mu \mathrm{L})$.

\subsection{Flexural strength, weibull parameters, and surface analyses}

Thirty specimens were prepared for each group. Two outliers of the infiltrated zirconia were disregarded in the analysis and analyzed separately.

To determine flexural strength, we placed each disc on a circular base with three metallic beads of $3.2 \mathrm{~mm}$ in diameter, equidistant from each other, forming a plane. A blunt tip, $1.6 \mathrm{~mm}$ in diameter, was attached to a testing machine (EMIC DL-1000, EMIC, São José dos Pinhais, PR, Brazil), and a load was applied (1000 Kgf) until fracture at a constant speed of $1 \mathrm{~mm} / \mathrm{min}$.

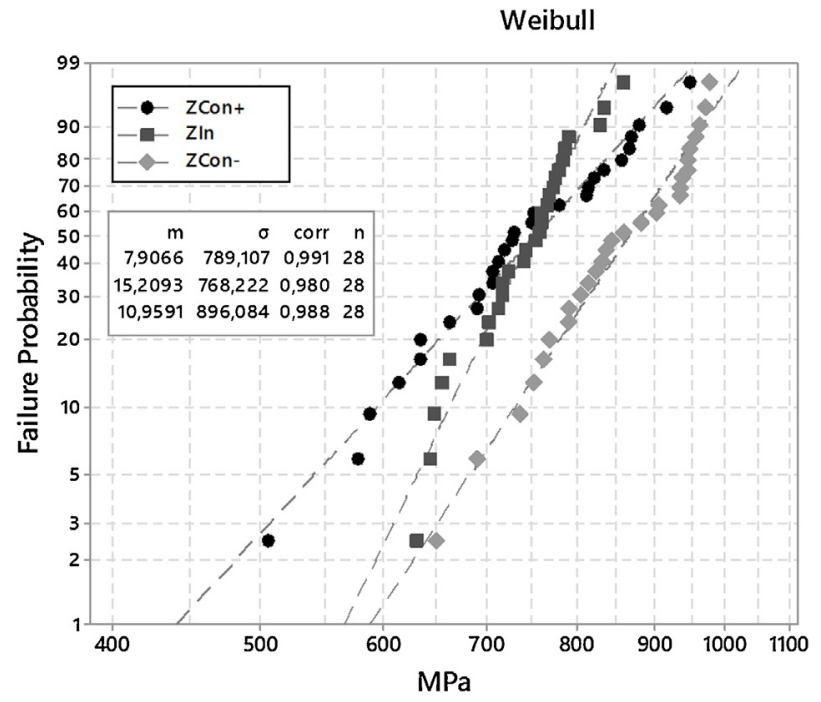

Fig. 1. Weibull plot of pure (ZCon-) zirconia, infiltrated zirconia (ZIn), and zirconia stored in water (ZCon +). The Weibull plot shows the steeper inclination of the line corresponding to the infiltrated group (higher Weibull modulus), with less dispersion of strength values than the positive and negative controls. The test for equal shape parameters $(P=0.001)$ showed that the moduli of the negative and infiltrated groups were not significantly different, but were both superior to the modulus of the positive control group. 
The biaxial flexural strength $(\sigma)(\mathrm{MPa})$ of the discs was obtained according to the ISO 6872 standard:

$\sigma=-0,2387 P(X-Y) / b^{2}$,

where: $P=$ load $(\mathrm{N}) ; X$ and $Y=$ are parameters related to a elastic property of the material [Poisson's ratio $(0.3)$ ] and the radii of the specimen and loading device $(\mathrm{mm})$; and $b=$ thickness of the specimen at the fracture origin in $\mathrm{mm}$.
The Weibull parameters, modulus (Shape parameter) and characteristic strength (Scale parameter), were obtained from the Maximum Likelihood estimation (Minitab 16, Minitab Inc, State College, PA, USA) (Fig. 1).

Topographic and fracture analyses were performed by stereomicroscopy (Discovery V20, Carl Zeiss, Jena, Germany) and scanning electron microscopy (SEM) (Inspect S50, FEI Company, Brno, Czech Republic).

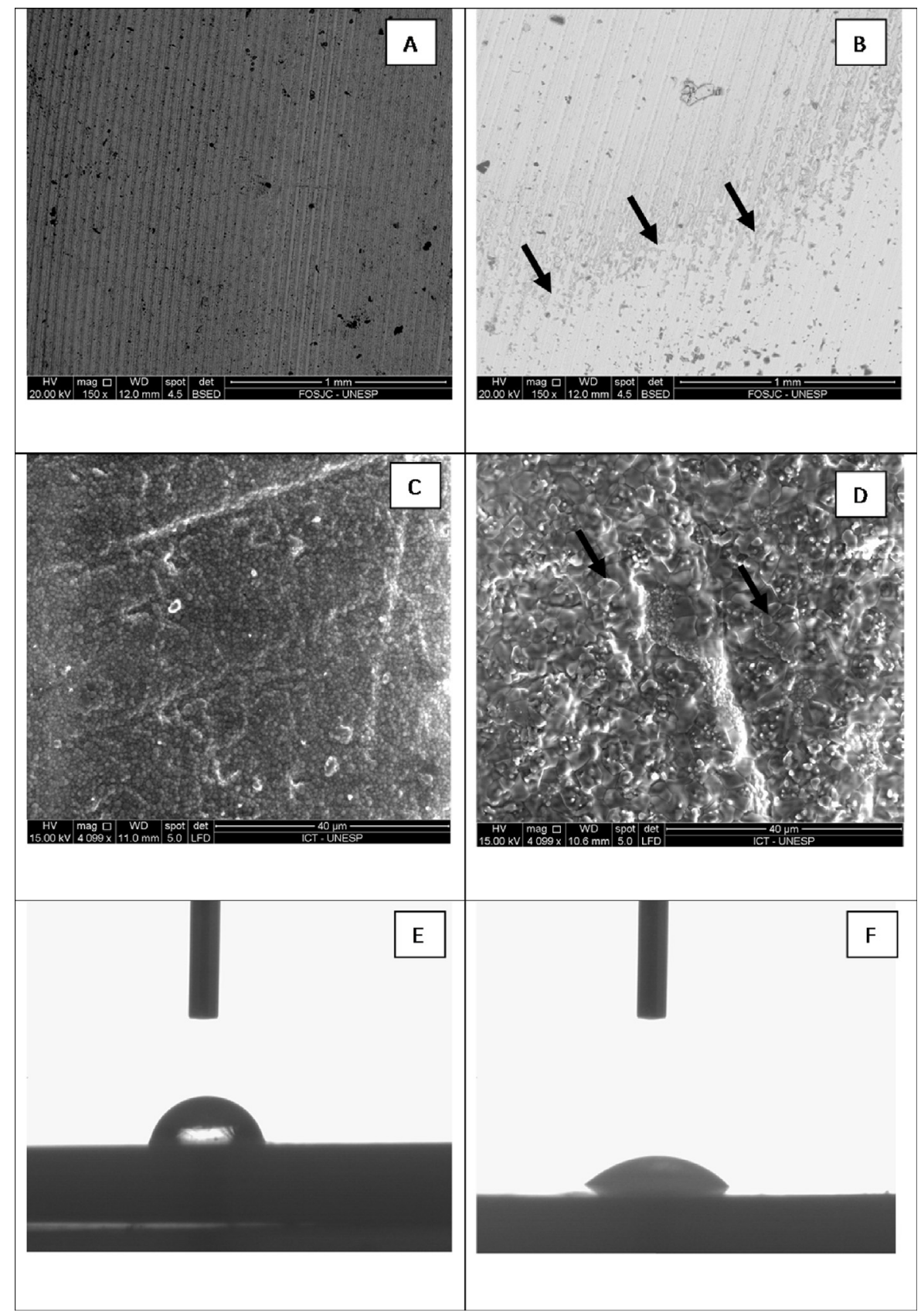

Fig. 2. Backscattered (A, B) and secondary (C, D) SEM and contact angle analysis of ZCon- (E) and ZIn (F) specimens. The charged areas indicate the deposition of silica, and the arrow indicates thicker deposition zones. In (C), one can see the zirconia grains, and in (D) the grains are permeated by the silica layer (arrows). The contact angles of pure (E) zirconia are higher than those of the infiltrated $(\mathrm{F})$ specimens, in which the water is more spread. 


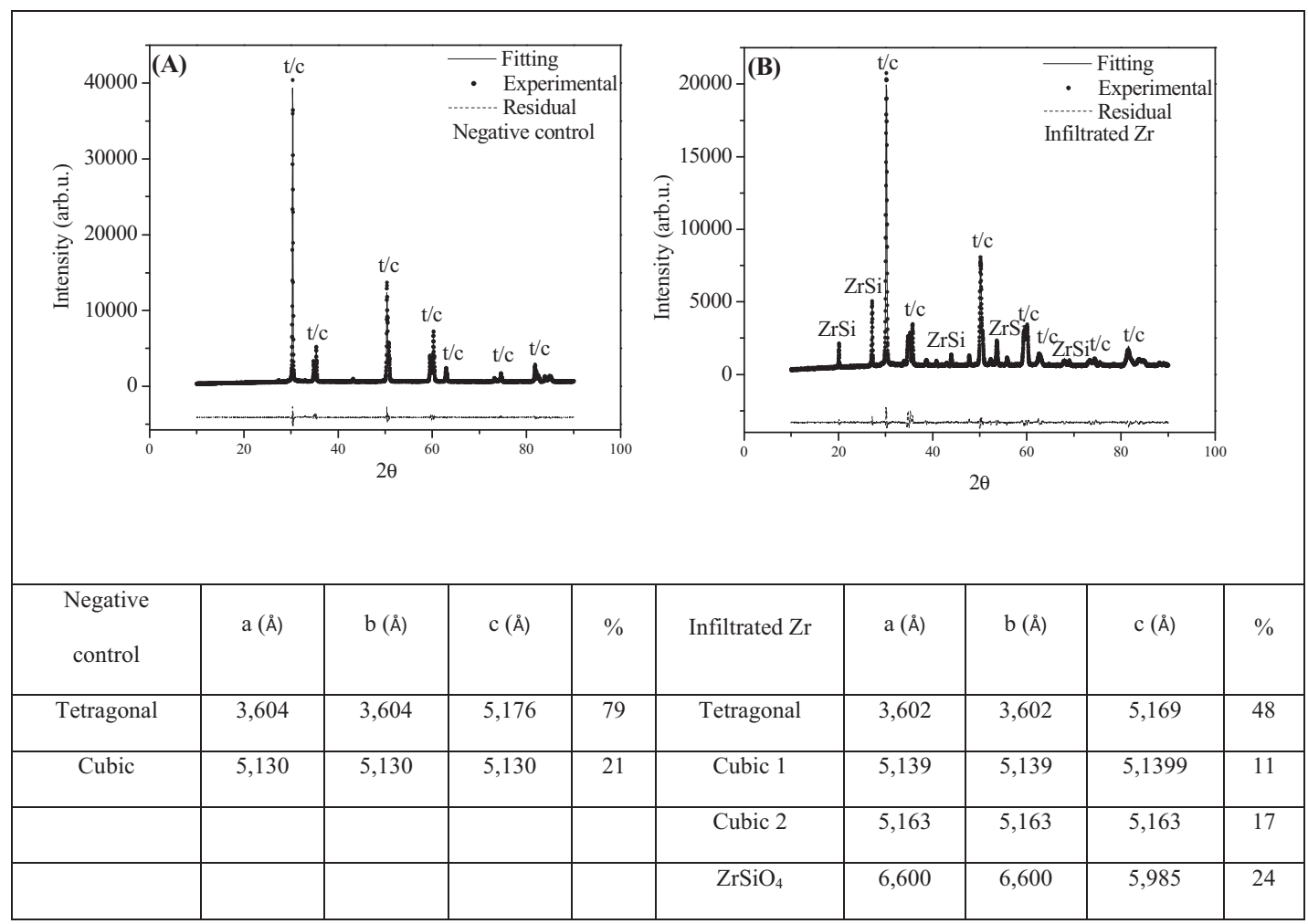

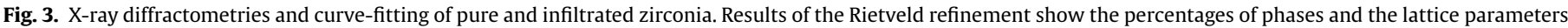
(A). The peaks signaled by $\mathrm{t} / \mathrm{c}$ correspond to tetragonal and cubic phases, whereas the $\mathrm{ZrSi}$ correspond to the $\mathrm{ZrSiO}_{4}$ composite.

A bond strength analysis was performed on square-shaped zirconia specimens. Specimens were divided in infiltrated and non-infiltrated groups (control). After sintering, the infiltrated groups were conditioned with $2 \% \mathrm{HF}$ acid for $10 \mathrm{~s}$. This acid concentration delivered the most favorable topography based on profilometry images of $2 \%, 5 \%$ and $10 \%$ of infiltrated specimens, after several exposition times (10s and 15s) (not shown here for brevity).

Specimens from both groups were then silanated with Monobond Plus or Monobond S (Ivoclar Vivadent, Schaan, Liechtenstein) and a resin cement cylinder ( $3 \mathrm{~mm}$ diameter and $6 \mathrm{~mm}$ height) (Variolink II, Ivoclar Vivadent, Schaan, Liechtenstein) was built up on each zirconia specimen. After photopolymerization for $40 \mathrm{~s}$, specimens were stored in distilled water for $24 \mathrm{~h}$ at $37^{\circ} \mathrm{C}$. Half of them were then tested in shear in a universal testing machine, while the other half was further subjected to thermalcycling $\left(5^{\circ}-55^{\circ} \mathrm{C}\right)$. After $6 \times 103$ cycles, the specimens were tested in shear and all fractured surfaces were analyzed in a stereomicroscope to determine the failure modes.

\section{Results}

The lowest and highest Weibull moduli belonged to $\mathrm{ZCon}^{+}$and ZIn groups, respectively (Fig. 1).

The silica infiltrated the bottom and top of the disc uniformly, but presented areas of increased deposition (Fig. 2A-D). The contact angles were inferior in the infiltrated specimens, which is a good indication of higher wettability and affinity to adhesive materials (Fig. 2E and F).
Fig. 3 shows that the infiltrated and negative control specimens presented peaks related to tetragonal and cubic zirconia; however, the former also presented peaks of zirconium silicate $\left(\mathrm{ZrSiO}_{4}\right)$. The specimens that were kept in water and the control group did not present phase changes; thus, only the infiltrated and the pure zirconia spectra are shown in Fig. 3. The Rietveld refinement identified the cubic and tetragonal phases, showing $79 \%$ of tetragonal and $21 \%$ of cubic phases.

Fig. 4 presents the fracture characteristics of all groups. The fracture origins were more prominent in the ZIn and ZCon ${ }^{-}$groups that in $\mathrm{ZCon}^{+}$.

Fig. 5 presents the Raman spectra of control and infiltrated specimens in which the ZIn fractured specimen shows preserved capacity for phase transformation.

The bond strength analysis showed lower bonding values and greater scattering of the data of non-infiltrated groups (Fig. 7). Non-infiltrated specimens that did not receive a layer of MDP could not survive after thermal cycling (TC). Table 1 displays the results of the infiltrated groups. The infiltrated groups treated with MDP presented the highest bond strength values before thermalcyling. Profilometry images showed the topography varied according to the surface treatment (Fig. 6). All failures were adhesive at the bonding interface.

\section{Discussion}

The Weibull analysis showed that the homogeneity of the new material was superior, due possibly to healing of surface flaws. Leaching of zirconia kept in water probably caused a variation in 

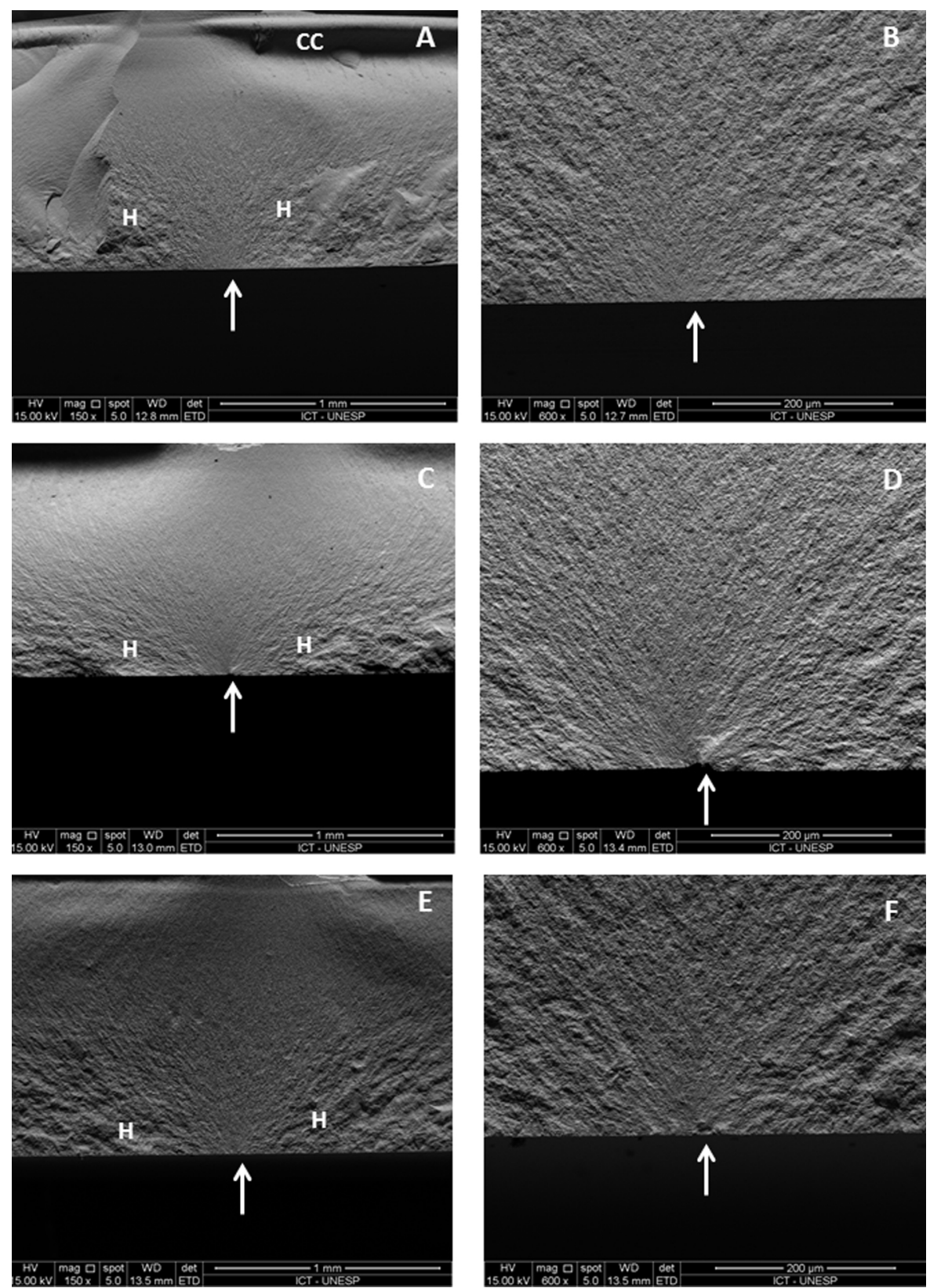

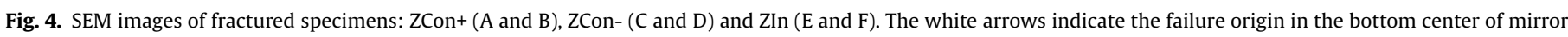

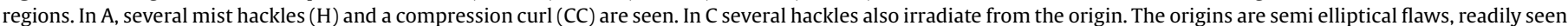
in $\mathrm{D}$ and $\mathrm{F}$, whereas in $\mathrm{B}$ no defect can be defined.

the severity of the defects on the surface, leading to a low Weibull modulus. Thus, less energy must have been needed to fracture $\mathrm{ZCon}^{+}$specimens.

The silica deposition also formed a sandwich of glass/zirconia/ glass layers, which share the characteristics of the graded zirconia described by Zhang et al. [22]. This type of material presents a gradation of elastic moduli toward the interior of the zirconia, which increases its load-bearing capacity and protects against low thermal degradation.

The increase in the wettability of ZIn turns this material into a good substrate for bonding resinous materials, treatable with HF and silanes. Moreover, the sol-gel infiltration presents the advantage of being a user-friendly method that does not impinge on the ceramic surface, as does air abrasion [23,24].

Silica infiltration was able to improve not only the immediate bond strength to resin cement, but also the long term bond strength after $6 \times 103$ thermal cycles. However, MDP primers have limited durability and that therefore explains the lower bond strengths between infiltrated zirconia and cement after thermacycling [12]. In spite of that, the bonding values were equivalent to the groups for which the infiltration was performed and no MDP was used, confirming that the 


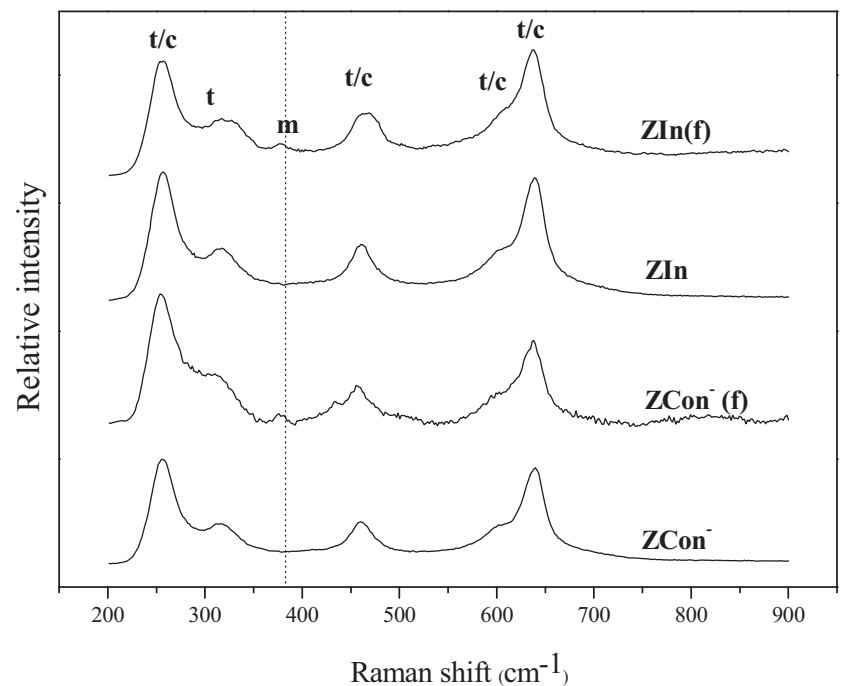

Fig. 5. Raman spectra of infiltrated and control zirconia of whole and fractured (f) specimens. Tetragonal zirconia $(\mathrm{t})$, tetragonal and cubic zirconia, monoclinic zirconia $(\mathrm{m})$.

mechanical interlocking is essential to preventing interfacial debonding.

The XRD peak analyses indicate that the silica diffused into the substrate and reacted with zirconia, forming an intermediate crystalline phase that tends to be more resistant to corrosion [25] because the pure silica gel infiltrated in the surface porosities is free of Sodium. But this needs further investigation.

It is important to highlight that the X-rays had limited penetration inside the material. Thus, heterogeneous materials, such as ceramic films, present peak intensities more representative of the film than the substrate. Because the Rietveld refinement is based upon the relative intensity of the peaks, the phase percentages are higher on the surface than in the bulk.

The infiltrated zirconia presented $24 \%$ of $\mathrm{ZrSiO}_{4}$ and $48 \%$ tetragonal zirconia. The modified zirconia also presented two types of cubic zirconia, types 1 and 2, which corresponded to 11 and 17\% of the material, respectively. Type 1 presents lattice parameters similar to those present in the control group; thus it can be said

Table 1

Bond strength data between zirconia and resin cement-Descriptive statistics [Mean, Standard Deviation (SD), and Coefficient of Variation (CV)] and Tukey's test of the infiltrated groups, with and without MDP and in the presence or in the absence of thermalcycling (TC). Different superscript letters mean statistically significant difference.

\begin{tabular}{llrlr}
\hline Infiltrated groups & $\mathrm{n}$ & Mean & SD & \multicolumn{1}{c}{ CV } \\
\hline MDP and TC & 10 & $3.62^{\mathrm{B}}$ & 3.79 & 104.78 \\
MDP no TC & 10 & $11.64^{\mathrm{A}}$ & 5.31 & 45.63 \\
No MDP and TC & 10 & $3.80^{\mathrm{B}}$ & 9.71 & 255.29 \\
No MDP no TC & 10 & $3.31^{\mathrm{B}}$ & 2.67 & 80.80 \\
\hline
\end{tabular}

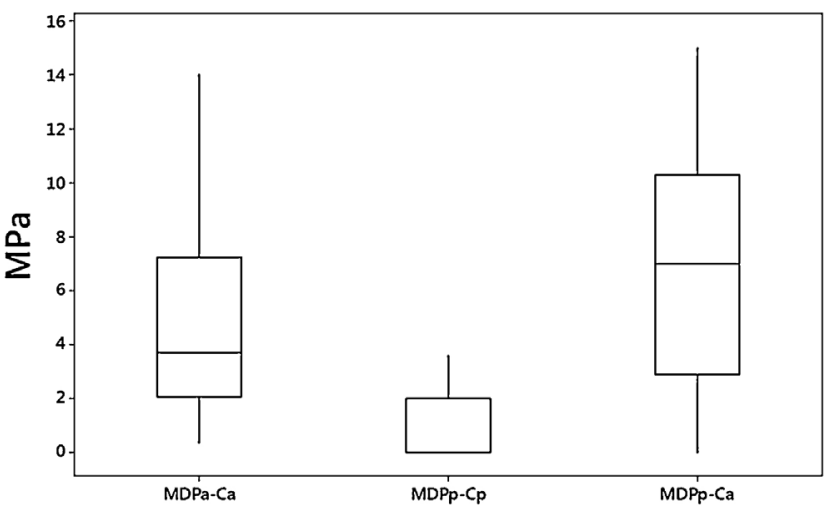

Fig. 6. Box-plots of the non-infiltrated groups, where $\mathrm{a}=\mathrm{absent} ; \mathrm{Ca}=$ cycling absent; $\mathrm{p}=$ present; $\mathrm{Cp}=$ cyling present. After thermalcycling, the bond strengths were lower and presented greater variability than the initial bond strengths. The groups that did not receive an MDP coating did not survive thermalcycling and could not be tested.

that this cubic zirconia is the same type as present in the original substrate.

$\mathrm{ZrSiO}_{4}$ and type 2 cubic zirconia are the main components of the film layer. This is possible because, at $1550^{\circ} \mathrm{C}$, silica on the zirconia surface can suffer a reaction on its solid state, resulting in zirconia silicate [26]. Besides $\mathrm{ZrSiO}_{4}$, the formation of another type of cubic zirconia occurs concurrently, which can affect the final properties of the material. This means that phase transformation that makes zirconia tougher than other ceramics [27-30], which is manifested in the phase changing from tetragonal to monoclinic during mechanical stimuli [30], is not present in cubic zirconia [31]. The increase in cubic phase thus stabilizes zirconia.

The Raman spectra of intact and fractured infiltrated and control specimens presented the peaks related to tetragonal and cubic zirconia $\left(250,315,460,600\right.$, and $\left.640 \mathrm{~cm}^{-1}\right)$ [30]. The only difference was the peak corresponding to monoclinic zirconia in the fractured specimens. Thus, one can suggest that the toughening transformation still occurs in the infiltrated zirconia and preservers its high toughness, but this needs more clarification. In spite of that, the mechanical properties of the infiltrated zirconia are slightly changed compared with those of pure zirconia, probably as a result of cubic phase formation.

The present study showed the sol-gel method was an efficient and simple way to increase the structural homogeneity and wettability of zirconia. Silica infiltration formed an intermediate crystalline phase and not only a juxtaposed silica layer. Additionally, the adhesion performance with $2 \%$ hydrofluoric acid for $5 \mathrm{~s}$ and MDP primer increased the bond strength to resin cement.

\section{Acknowledgement}

This study did not receive funding from external sources and had no conflicts of interest. 


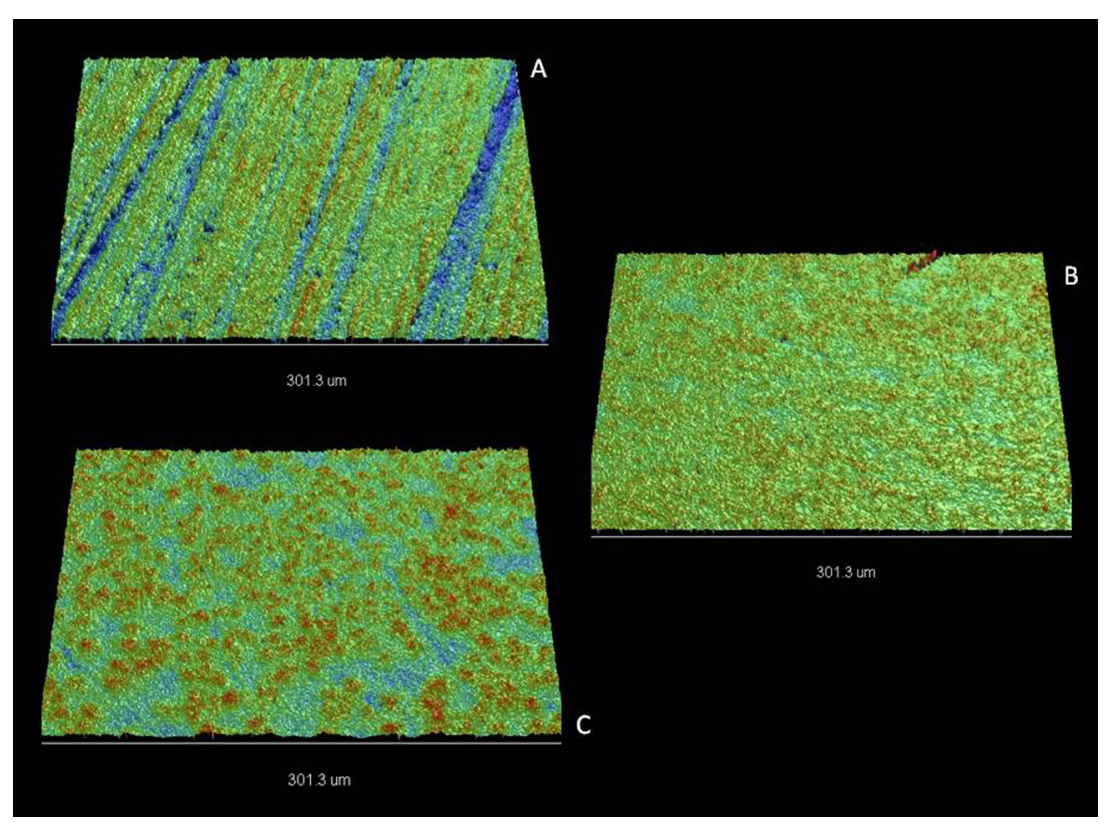

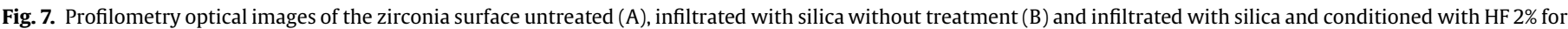
$10 \mathrm{~s}(\mathrm{C})$.

\section{References}

[1] T. Miyazaki, T. Nakamura, H. Matsumura, S. Ban, T. Kobayashi, Current status of zirconia restoration, J. Prosthodont. Res. 57 (4) (2013) 236-261.

[2] B.I. Ardlin, Transformation-toughened zirconia for dental inlays, crowns and bridges: chemical stability and effect of low-temperature aging on flexural strength and surface structure, Dent. Mater. 18 (8) (2002) 590-595.

[3] C.G. Figueiredo-Pina, A. Monteiro, M. Guedes, A. Maurício, A.P. Serro, A. Ramalho, C. Santos, Effect of feldspar porcelain coating upon the wear behavior of zirconia dental crowns, Wear 297 (2013) 872-877.

[4] I. Denry, J.R. Kelly, State of the art of zirconia for dental applications, Dent. Mater. 24 (3) (2008) 299-307.

[5] M. Guazzato, M. Albakry, S.P. Ringer, M.V. Swain, Strength, fracture toughness and microstructure of a selection of all-ceramic materials. Part II. Zirconiabased dental ceramics, Dent. Mater. 20 (5) (2004) 449-456.

[6] C. Mochales, A. Maerten, A. Rack, P. Cloetens, W.D. Mueller, P. Zaslansky, C. Fleck, Monoclinic phase transformations of zirconia-based dental prostheses, induced by clinically practiced surface manipulations, Acta Biomater. 7 (7) (2011) 2994-3002.

[7] J.Y. Thompson, B.R. Stoner, J.R. Piascik, R. Smith, Adhesion/cementation to zirconia and other non-silicate ceramics: where are we now? Dent. Mater. 27 (1) (2011) 71-82.

[8] R. Curtis, A.J. Wright, G.J. Fleming, The influence of surface modification techniques on the performance of a Y-TZP dental ceramic, J. Dent. 34 (2006) 195.

[9] N. Mahmoodi, T. Hooshmand, S. Heidari, K. Khoshro, Effect of sandblasting, silica coating, and laser treatment on the microtensile bond strength of a dental zirconia ceramic to resin cements, Lasers Med. Sci. 31 (2) (2016) 205 211.

[10] B. Yang, A. Barloi, M. Kern, Influence of air-abrasion on zirconia ceramic bonding using an adhesive composite resin, Dent. Mater. 26 (1) (2010) 44-50.

[11] P. Magne, M.P.G. Paranhos, L.H. Burnett Jr., New zirconia primer improves bond strength of resin-based cements, Dent. Mater. 26 (2010) 345-352.

[12] M. Kern, Bonding to oxide ceramics-laboratory testing versus clinical outcome, Dent. Mater. 31 (2015) 8-14.

[13] A. Casucci, F. Monticelli, C. Goracci, C. Mazzitelli, A. Cantoro, F. Papacchini, M. Ferrari, Effect of surface pre-treatments on the zirconia ceramic-resin cement microtensile bond strength, Dent. Mater. 27 (10) (2011) 1024-1030.

[14] C. Larsson, A. Wennerberg, The clinical success of zirconia-based crowns: a systematic review, Int. J. Prosthodont. 27 (2014) 33-43.

[15] R. Janda, J.F. Roulet, M. Wulf, H.J. Tiller, A new adhesive technology for allceramics, Dent. Mater. 19 (6) (2003) 567-573.
[16] T.A. Valentino, G.A. Borges, L.H. Borges, J.A. Platt, L. Correr-Sobrinho, Influence of glazed zirconia on dual-cure luting agent bond strength, Oper. Dent. 37 (2) (2012) 181-187.

[17] A. Vanderlei, M.A. Bottino, L.F. Valandro, Evaluation of resin bond strength to yttria-stabilized tetragonal zirconia and framework marginal fit: comparison of different surface conditionings, Oper. Dent. 39 (1) (2014) 50-63.

[18] J.R. Queiroz, M. Massi, L. Nogueira Jr., A.S. Sobrinho, M.A. Bottino, M. Ozcan, Silica-based nano-coating on zirconia surfaces using reactive magnetron sputtering: effect on chemical adhesion of resin cements, J. Adhes. Dent. 15 (2) (2013) 151-159.

[19] M.N. Aboushelib, Evaluation of zirconia/resin bond strength and interface quality using a new technique, J. Adhes. Dent. 13 (2011) 255-260.

[20] A. Casucci, E. Osorio, R. Osorio, F. Monticelli, M. Toledano, C. Mazzitelli, M. Ferrarri, Influence of different surface treatments on surface zirconia frameworks, J. Dent. 37 (11) (2009) 891-897.

[21] Y. Zhang, M. Li, Optimization of ceramic strength using elastic gradients, Acta Mater. 57 (9) (2012) 2721-2729.

[22] Y. Zhang, H. Chai, J.J.-W. Lee, B.R. Lawn, Chipping resistance of graded zirconia ceramics for dental crowns, J. Dent. Res. 91 (3) (2012) 311-315.

[23] R.O. Souza, L.F. Valandro, R.M. Melo, J.P. Machado, M.A. Bottino, M. Ozcan, Airparticle abrasion on zirconia ceramic using different protocols: effects on biaxial flexural strength after cyclic loading, phase transformation and surface topography, J. Mech. Behav. Biomed. Mater. 26 (2013) 155-163.

[24] Y. Zhang, B.R. Lawn, E.D. Rekow, V.P. Thompson, Effect of sandblasting on the long-term performance of dental ceramics, J. Biomed. Mater. Res. B 71 (B) (2004) 381-386.

[25] I. Denry, How and when does fabrication damage adversely affect the clinical performance of ceramic restorations? Dent. Mater. 29 (1) (2013) 85-96.

[26] A. Sharif, P.H. Imamura, T.E. Mitchell, M.L. Mecartney, Control of grain growth using intergranular silicate phases in cubic yttria stabilized zirconia, Acta Mater. 46 (11) (1998) 3863-3872.

[27] S. Deville, L. Gremillard, J. Chevalier, G. Fantozzi, A critical comparison of methods for the determination of the aging sensitivity in biomedical grade yttria-stabilized zirconia, J. Biomed. Mater. Res. B 72 (2) (2005) 239-245.

[28] I. Denry, J.A. Holloway, Ceramics for dental applications: a review, Materials 3 (1) (2010) 351-368.

[29] V. Lughi, V. Sergo, Low temperature degradation - aging - of zirconia: a critical review of the relevant aspects in dentistry, Dent. Mater. 26 (8) (2010) 807-820.

[30] P. Pittayachawan, A. McDonald, A. Young, J.C. Knowles, Flexural strength, fatigue life, and stress-induced phase transformation study of Y-TZP dental ceramic, J. Biomed. Mater. Res. B 88 (2) (2009) 366-377.

[31] M.B. Ponnuchamy, A.S. Gandhi, Lattice expansion and contraction in nanocrystalline yttria-stabilized zirconia powders, Scr. Mater. 83 (2014) 21 24. 